\title{
Short-term changes in substance use among problematic alcohol and drug users from a general population sample
}

\author{
Kristina Sinadinovic ${ }^{1,2}$, Peter Wennberg ${ }^{3,4}$, and Anne H. Berman ${ }^{1,2}$ \\ ${ }^{1}$ Karolinska Institutet, Department of Clinical Neuroscience, Center for Psychiatric Research, Stockholm, Sweden \\ ${ }^{2}$ Stockholm Center for Dependency Disorders, Stockholm, Sweden \\ ${ }^{3}$ Stockholm University, Center for Social Research on Alcohol and Drugs (SoRAD), Stockholm, Sweden \\ ${ }^{4}$ Karolinska Institutet, Department of Public Health, Division of Social Medicine, Norrbacka, Stockholm, Sweden
}

\section{Abstract}

Aims: To investigate 12-month changes among individuals with baseline problematic use of alcohol or illicit drugs, by gender, age, initial level of substance use, and administration method (Internet or Interactive Voice Response, IVR).

Design: 1,861 individuals from a random population sample were screened for problematic alcohol and illicit drug use. Individuals screening positive were followed up after 12 months.

Setting: The Swedish general population.

Participants: 423 individuals with baseline problematic alcohol (women: AUDIT $\geq 6$, men: AUDIT $\geq 8$ ) or drug use (both genders: DUDIT $\geq 1$ ).

Measures: AUDIT, DUDIT, AUDIT-C and DUDIT-C scores.

Findings: AUDIT scores decreased from 10.43 to 8.62; among 239 participants with baseline problematic alcohol use, $34.3 \%$ no longer had problematic use at follow-up. Total DUDIT scores decreased from 4.92 to 2.33; among 51 participants with baseline problematic drug use, $60.8 \%$ reported no illicit drug use at follow-up. AUDIT and DUDIT scores decreased more for individuals who at baseline had harmful problematic use, compared to those who had hazardous baseline use. Within-group effect sizes for AUDIT and DUDIT scores varied between 0.29 and 0.69 (Cohen's $d$ ).

Conclusions: Data on short-term change in problematic substance use in a random general population sample could constitute a reference point for comparisons for uncontrolled treatment studies.

Alcohol use is one of the world's leading risk factors for disability-adjusted life years and death, especially in middle- and high-income countries. Worldwide, $3.6 \%$ of all deaths can be attributable to the use of alcohol and $0.4 \%$ to illicit drug use. In addition, $4.4 \%$ of disability-adjusted life years are caused by alcohol use and $0.9 \%$ by illicit drug use, with a high social cost as a consequence (WHO, 2009). One of several effective strategies for reducing alcoholrelated harm is early intervention and treatment (WHO, 2007), a strategy which might also be applicable to the context of illicit drugs.

Estimating effect sizes for different intervention and treatment methods is a considerable challenge. One of the difficulties is that there is great heterogeneity between studies in terms of results, research design, participant characteristics and control groups. Consequently, the method by which studies are compared strongly influences apparent comparative effect sizes. Literature reviews showed that the effect sizes of a specific treatment are, in some studies, compared to effect sizes for other treatments; in other studies, effect sizes derive from comparison to a control group that did not receive any intervention; and in yet another type of study, effect sizes reported from investigations without control groups concern within-group effects rather than between-group effects. In addition, the severity of substance use differs widely from study to study. This inconsistency makes it difficult to draw conclusions regarding an overall effect size for a specific treatment (The National Board of Health and Welfare, 2006; The Swedish Council on Technology Assessment in Health Care, 2001a, 2001b). As an illustration, recommendations regarding evidence-based early intervention and treatment, given in the Swedish National Board of Health and Welfare 2007 National Guidelines for Addiction Treatment (The National Board of Health and Welfare, 2007), showed weighted effect sizes from 0.20 to 0.54 for methods reducing problematic alcohol use (The

Correspondence: Kristina Sinadinovic, Ph.D., Karolinska Institutet, Department of Clinical Neuroscience, Center for Psychiatric Research, Stockholm, Norra Stationsgatan 69, $7^{\text {th }}$ floor, SE-113 64 Stockholm, Sweden. Telephone: 08- 123457 35. FAX: 08-524 830 49. E-mail: kristina.sinadinovic@ki.se

Keywords: Screening; general population; natural variation; alcohol; illicit drugs; spontaneous remission 
Swedish Council on Technology Assessment in Health Care, 2001a) and from -0.68 to 2.63 for methods reducing problematic drug use (The Swedish Council on Technology Assessment in Health Care, 2001b). In the context of treatment, an effect size of 0.20 is considered small, 0.50 medium and 0.80 and above as large (Cohen, 1988).

Previous research has shown that there is a wide range of different types of treatment services that people can use to change problematic substance use (Raistrick, Heather, \& Godfrey, 2006; The National Board of Health and Welfare, 2007). However, the vast majority of those with problematic alcohol use (Blomqvist, 1996, 1999, 2002; Cunningham, 2005; Cunningham \& Blomqvist, 2006; Cunningham \& Breslina, 2004), as well as those using illegal drugs (Blomqvist, 1996, 2002; Walters, 2000), change their problematic use without any professional help. In addition, self-help Internet-based interventions (Cunningham, Wild, Cordingley, van Mierlo, \& Humphreys, 2009; Hester, Delaney, Campbell, \& Handmaker, 2009; Kypri et al., 2009; Riper et al., 2009; Sinadinovic, Berman, Hasson, \& Wennberg, 2010) and other kinds of self-help materials (Cunningham, Humphreys, Koski-Jännes, \& Cordingley, 2005; KoskiJännes \& Cunningham, 2001) have been shown to have a positive effect on changing problematic alcohol use. Furthermore, problematic alcohol users often tend to use more than just one method to change their use (Blomqvist, Cunningham, Wallander, \& Collin, 2007; Cunningham \& Blomqvist, 2006), suggesting it is difficult to isolate one specific factor that leads to such change. Similar recovery patterns are to be found for problematic drug users (Blomqvist, 1996; Walters, 2000).

Given the wide range of mixed treatments as well as the high proportion of individuals changing their alcohol use without professional help, we identified a need to measure overall short-term changes in problematic alcohol and illicit drug use at a general population level, irrespective of any treatment received or recovery strategies used. We suggest that the level of such change might serve as a future population-based reference point against which effect sizes of different treatments could be measured for a fair comparison, and as a reference point for uncontrolled treatment studies.

\section{Aims}

The aim of this study was to investigate 12-month changes in alcohol and drug use among individuals with baseline problematic alcohol or illicit drug use. The effects of gender, age, initial level of problematic substance use and questionnaire administration method (Internet or Interactive Voice Response) are analyzed.

\section{Method}

\section{Baseline Population Screening of Problematic Alcohol and Illicit Drug Use}

In 2008, 5,000 individuals (aged 16 to 80 years) were randomly selected from the Swedish general population and contacted via post. In total, 1,861 individuals (53.3\% women and $46.7 \%$ men) chose to participate in the baseline study and were screened for problematic alcohol and illicit drug use using Internet and Interactive Voice Response (IVR) versions of the AUDIT (Alcohol Use Disorders Identification Test; Saunders, Aasland, Babor, De La Fuente, \& Grant, 1993) and the DUDIT (Drug Use Disorders Identification Test; Berman, Bergman, Palmstierna, \& Schlyter, 2005). Both these tests were recommended in 2007 by the Swedish National Board of Health and Welfare for identification of risky use of alcohol and illicit drugs. For this study, we selected a sampling pool consisting of 423 individuals from among the 1,861 individuals in our population sample. The sampling pool included all individuals who, at baseline, showed problematic alcohol use (AUDIT $\geq 6$ for women and $\geq 8$ for men) or problematic illicit drug use (DUDIT $\geq$ 1 for both men and women). No significant differences in alcohol and drug use were found between those who participated in the study via Internet and those who participated via IVR (Sinadinovic, Berman, \& Wennberg, 2011).

\section{Procedure}

At the population screening in 2008, all individuals in the sampling pool were informed that they might be contacted again in order to investigate changes in their alcohol and illicit drug use over time. Twelve months later, all 423 individuals who had at least hazardous alcohol or drug use at the baseline screening (51.3\% women and $48.7 \%$ men) were again contacted via post and invited to participate in a follow-up study. Individuals were offered the same administration mode that they had used before, whether this was Internet or IVR. The sub-group of those who had been offered a choice between Internet and IVR at the initial screening was offered the same choice at follow-up, irrespective of which administration mode the individuals had chosen for the initial screening. On September 1, 2009, the first invitation letter for the follow-up was posted, with a unique log-in code for each participant. Two reminders were sent out at intervals of three weeks. The participants could respond to the survey until January 31, 2010. In total, 33 individuals had moved away during the follow-up period and did not receive the letters sent out from the research team, resulting in a total sampling pool of 390 individuals from the Swedish general population who had at least hazardous alcohol or illicit drug use.

\section{Response Rate}

The overall response rate for the follow-up was $67.9 \%$ ( $n=$ 265; $50.2 \%$ women and $49.8 \%$ men). Nominal differences in response rates by administration mode, shown in Table 1 , are not statistically significant. 
Table 1

Participant flow by response mode $(n=423)$

\begin{tabular}{lcccc}
\hline & \multicolumn{3}{c}{ Response mode } & \\
\cline { 2 - 5 } & Internet & IVR & $\begin{array}{c}\text { Respondent choice } \\
\text { Internet/IVR }\end{array}$ & Total \\
\hline Initial sampling pool & 167 & 148 & 108 & 423 \\
Incorrect addresses & 12 & 11 & 10 & 33 \\
Final sampling pool & 155 & 137 & 98 & 390 \\
& & Cumulative $\boldsymbol{n}$ & 148 \\
Response without reminder & 60 & 50 & 38 & 223 \\
After one reminder & 91 & 76 & 56 & 265 \\
After two reminders & 111 & 85 & 69 & $68.0 \%$ \\
Response rate & & $62.0 \%$ & $70.4 \%$ & \\
\hline
\end{tabular}

${ }^{a}$ The minimum criterion for being included in the response rate was to fill out the AUDIT.

Of the 13 individuals who were offered a choice of administration mode and participated in the initial screening via IVR, six changed their response mode to Internet in the follow-up. Of the 56 individuals who were offered a choice of administration mode and participated in the initial screening via the Internet, only two changed their response mode to IVR in the follow-up. Sampling pool participants who did not respond to the follow-up study invitation are described in comparison to respondents in the Results section below.

\section{Outcome Measures}

The outcome measures were as follows: (1) Total AUDIT score, as a summarized measure of alcohol use including alcohol consumption and alcohol-related problems;(2) Total AUDIT score for the first three AUDIT questions, as a measure of alcohol consumption only, hereafter referred to as the AUDIT-C (Bush, Kivlahan, McDonell, Fihn, \& Bradley, 1998); (3) Problematic alcohol use, defined as a total AUDIT score of 6 or more for women and 8 or more for men; (4) Total DUDIT score, as a summarized measure of illicit drug use including illicit drug consumption and drug-related problems; (5) Total DUDIT score for the first four DUDIT questions, as a measure of illicit drug consumption only, hereafter referred to as the DUDIT-C; (6) Problematic illicit drug use, defined as a total DUDIT score of at least 1 for both genders.

\section{Definitions}

The terms hazardous use, harmful use, and probable dependence were defined in this article based on the total AUDIT score for alcohol and on the total DUDIT score for drugs, where higher scores indicate more severe substance use. Hazardous alcohol use was defined for men as scoring between 8 and 15 points and for women as scoring between 6 and 15 points on the AUDIT. Harmful alcohol use for both men and women was defined as scoring between 16 and 19 points, while probable dependence for both genders was defined as scoring 20 points or more.
Hazardous drug use was defined for men as scoring between 1 and 5 points and for women as scoring just 1 point on the DUDIT. Harmful drug use was defined for men as scoring between 6 and 24 points and for women as scoring between 2 and 24 points. Probable dependence was defined for both genders as scoring 25 points or more. All cut-off scores for both AUDIT and DUDIT are based on the officially established cut-off scores for these instruments in Sweden, taken from the published Swedish AUDIT and DUDIT manual (Berman, Wennberg, \& Källmén, 2012). In this article, the term problematic alcohol or drug use includes hazardous and harmful use, as well as probable dependence.

\section{Statistics}

Descriptive statistics-frequencies, means and standard deviations - were used to describe characteristics of the sample and of the non-respondents as well as participants' and non-respondents' alcohol and drug use at baseline. Chi-square tests were used to test the differences in proportions and prevalence of problematic substance use, while means were compared using independent $t$-tests. Changes in total AUDIT and DUDIT scores as well as in AUDIT-C and DUDIT-C scores were measured and tested by repeated measures ANOVA. Within-group effect sizes were measured using Cohen's $d$ with pooled standard deviations.

The study was positively reviewed by the Stockholm Regional Ethical Vetting Board (no. 2008/308-31/5) and carried out according to the Law (2003:460) on vetting the ethics of research involving humans. All data collection was carried out in accordance with the Personal Data Act (1998:204). 


\section{Results}

\section{Sample Characteristics}

In total, $50.2 \%(n=133)$ of the participants in the one-year follow-up were women and $49.8 \%(n=132)$ were men. The mean age for the sample was 40.6 years $(S D=16.6)$ with no significant differences between the genders. In total, $24.5 \%$ of the individuals in the sample $(n=65)$ were classified as 18 to 24 years old, $18.1 \%(n=48)$ were 25 to 34 years old, $48.7 \%(n=129)$ were 35 to 64 years old and $8.7 \%(n=23)$ were 65 to 81 years old.

\section{Non-respondents}

The non-response rate among women was $31.8 \%$ and among men $32.3 \%$, a non-significant gender difference. Non-respondents were, however, younger than participants at follow-up. The mean age for non-responding women was 33.1 years $(S D=16.2)$, significantly lower than the mean age for women participating in the follow-up (39.6; $t$ $=-2.52 ; d f=193 ; p=0.01)$. The corresponding age for non-responding men was $36.5(S D=17.0)$, also significantly lower than that for responding men (41.6; $t=$ -2.03; $d f=193 ; p=0.04$ ).

No significant differences between respondents' and nonrespondents' alcohol and drug use at baseline were found (AUDIT: non-respondents, 10.79; one-year follow-up participants, 10.43; proportions of hazardous alcohol use, harmful use, and probable dependence: non-respondents, $83.5 \%, 10.4 \%$ and $6.1 \%$; participants, $88.7 \%, 6.3 \%$ and 5.0\%; DUDIT: non-respondents, 6.32; participants 4.92; proportions of hazardous and harmful drug use: non- respondents, $41.9 \%$ and $58.1 \%$; participants, $41.2 \%$ and $58.8 \%)$.

\section{Changes in Problematic Alcohol Use Over 12 Months}

Over a 12-month follow-up period, a significant decrease in the total AUDIT score was found for both men and women in the sample of problematic alcohol users. The total AUDIT mean score for women decreased from 9.28 at the baseline to 7.12 at the 12-month follow-up. For men, the total AUDIT score decreased from 11.55 to 10.10 during the same period $(F=36.90 ; d f=1 ; p<0.001)$. Even though men's AUDIT scores were significantly higher than women's at follow-up ( $F=25.29 ; d f=1 ; p<0.001)$, the decline in men's and women's AUDIT scores was about the same for both genders.

The same results were found for the AUDIT-C. The AUDIT-C score decreased from 5.36 to 4.71 for women and from 6.36 to 5.98 for men $(F=21.03$; $d f=1 ; p<$ 0.001). There was a significant difference in alcohol consumption between men and women $(F=35.07$; $d f=1$; $p<0.001$ ) but the decrease over time was about the same in both groups. In both the AUDIT-C scores and the total AUDIT scores, no interaction effects were found for time and age, but there was an interaction effect for time and level of problematic alcohol use (AUDIT), as well as alcohol consumption (AUDIT-C). As shown in Figures 1a and $1 \mathrm{~b}$, the decline in both total AUDIT scores $(F=14.47$; $d f=2 ; p<0.001)$ and the AUDIT-C scores $(F=3.67 ; d f=$ $2 ; p=0.027$ ) was significantly larger for individuals with harmful use and probable dependence.

\section{Figure 1a}

Changes in total AUDIT scores by categories of problematic alcohol use

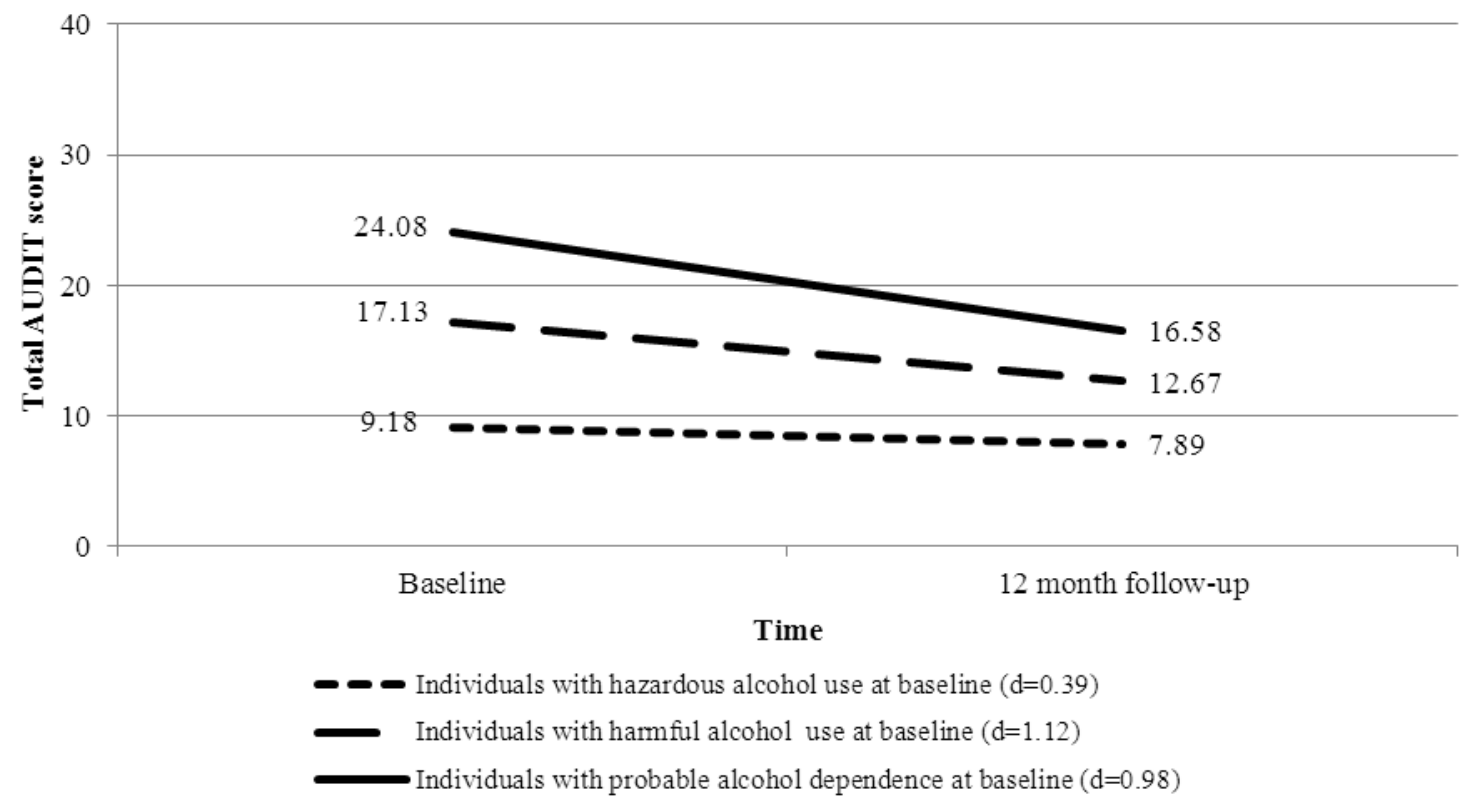




\section{Figure 1b}

\section{Changes in total AUDIT-C scores by categories of problematic alcohol use}

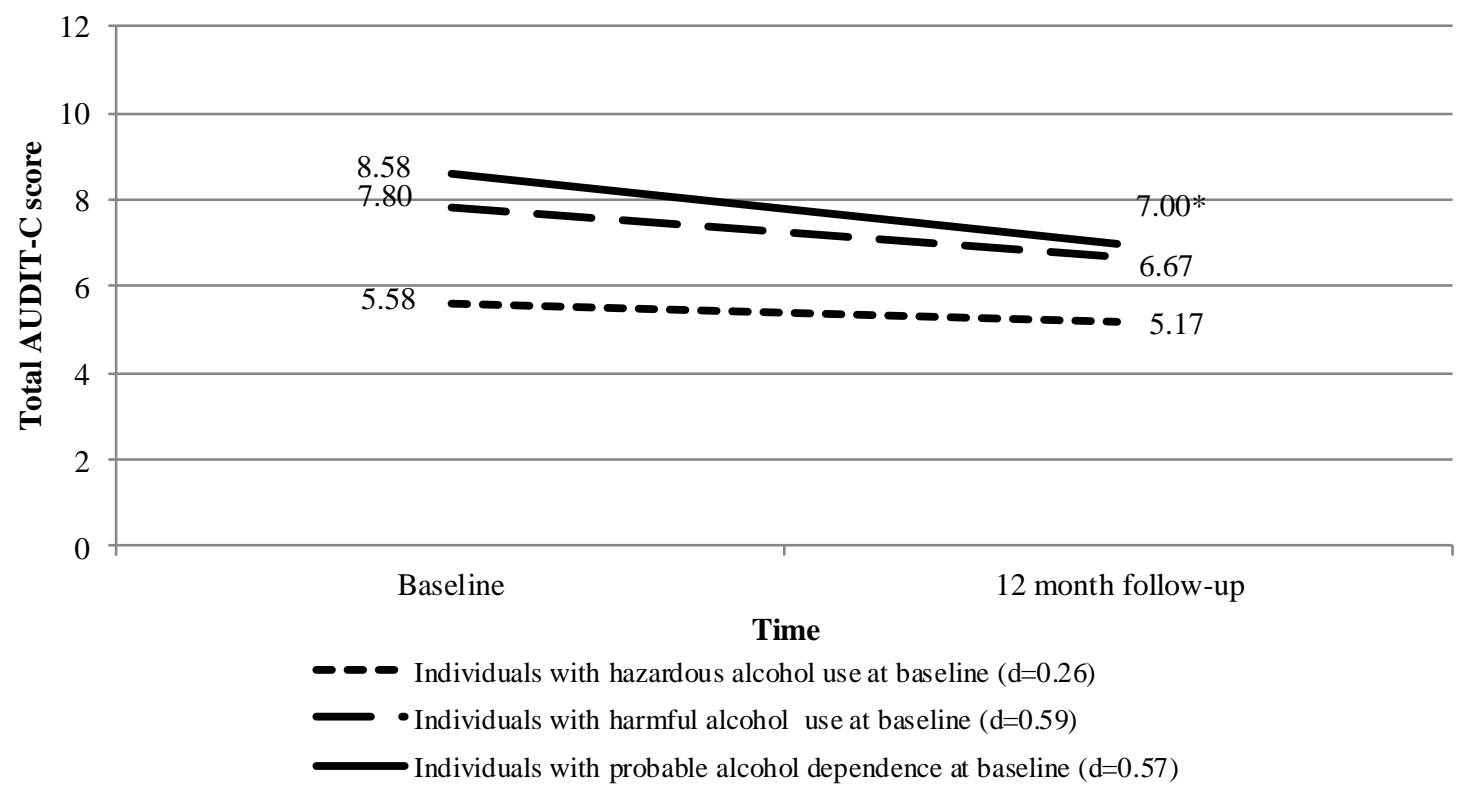

$* p<0.05$

Table 2

Changes in categories of problematic alcohol use over 12 months $(n=239)$

\begin{tabular}{|c|c|c|c|c|c|}
\hline \multirow[b]{2}{*}{$\begin{array}{l}\text { Baseline category of } \\
\text { problematic alcohol use }\end{array}$} & \multicolumn{4}{|c|}{ Follow-up category of problematic alcohol use } & \multirow[b]{2}{*}{ Total } \\
\hline & $\begin{array}{l}\text { No problematic } \\
\text { alcohol use }\end{array}$ & $\begin{array}{l}\text { Hazardous } \\
\text { alcohol use }\end{array}$ & $\begin{array}{l}\text { Harmful alcohol } \\
\text { use }\end{array}$ & $\begin{array}{l}\text { Probable alcohol } \\
\text { dependence }\end{array}$ & \\
\hline Hazardous alcohol use & $78(36.8 \%)$ & $127(59.9 \%)$ & $5(2.4 \%)$ & $2(0.9 \%)$ & $212(100.0 \%)$ \\
\hline Harmful alcohol use & $2(13.3 \%)$ & 7 (46.7\%) & $4(26.7 \%)$ & $2(13.3 \%)$ & $15(100.0 \%)$ \\
\hline Probable alcohol dependence & $2(16.7 \%)$ & $2(16.7 \%)$ & $2(16.7 \%)$ & $6(50.0 \%)$ & $12(100.0 \%)$ \\
\hline Total & $82(34.3 \%)$ & $136(56.9 \%)$ & $11(4.6 \%)$ & $10(4.2 \%)$ & $239(100.0 \%)$ \\
\hline
\end{tabular}

The changes in both AUDIT and AUDIT-C scores did not differ by administration mode (Internet versus IVR).

The overall within-group effect size over 12 months for the total AUDIT score was $d=0.38$ and for the AUDIT-C score $d=0.29$.

As shown in Table 2, 34.3\% $(n=82)$ of the 239 participants with problematic alcohol use at baseline no longer had problematic alcohol use at follow-up (35.6\% of the women in the sample and $33.1 \%$ of the men; n.s.).

In contrast, a larger proportion of men that participated in the follow-up via Internet (39.3\%) no longer had problematic alcohol use at follow-up, compared to men that participated via IVR (18.9\%) $\left(\chi^{2}=4.82 ; d f=1 ; p=0.03\right)$. No such statistically significant difference by administration mode was found for women.

Looking at the changes within each category of problematic alcohol use, the proportion of those that no longer had problematic alcohol use after the 12-month follow-up period was $36.8 \%$ among those with baseline hazardous alcohol use, $13.3 \%$ among those with baseline harmful use, and $16.7 \%$ among those with baseline probable dependence on alcohol. The relatively large nominal differences between the groups were not statistically significant. 


\section{Changes in Problematic Drug Use Over 12 Months}

Over the 12-month follow-up period, a significant decrease in total DUDIT scores was also found among both men and women in the sample of problematic drug users. The total mean DUDIT score for women decreased from 4.89 at baseline to 1.93 at the 12-month follow-up. For men, the total DUDIT score decreased from 4.96 to 2.79 after 12 months $(F=13.36 ; d f=1 ; p=0.001)$. The apparent differences between men's and women's illicit drug use at baseline and follow-up were not statistically significant, nor was there any significant difference by gender for the decline in total DUDIT scores.

The same results were found for the DUDIT-C, where scores decreased from 3.00 to 1.15 for women and from 3.00 to 2.04 for men $(F=14.53 ; d f=1 ; p<0.001)$. Also here, there was no significant difference in illicit drug consumption between men and women, and the decline was also about the same in both groups.

No interaction effect for time and age was found, but the declines in both total DUDIT score $(F=10.25$; $d f=1 ; p=$ $0.002)$ and the DUDIT-C score $(\mathrm{F}=7.61$; $\mathrm{df}=1 ; \mathrm{p}=$
0.008) were significantly larger for individuals with harmful drug use than for those with hazardous drug use. Figures $2 \mathrm{a}$ and $2 \mathrm{~b}$ show more details.

The overall within-group effect sizes for both the total DUDIT score and for the DUDIT-C score were $d=0.69$.

A majority of individuals with problematic drug use at baseline were no longer using any illicit drugs at follow-up: 31 of the 51 participants had quit $(60.8 \%)$. A gender analysis indicated that $66.7 \%$ of the women and $54.2 \%$ of the men with baseline problematic drug use had quit using illicit drugs at follow-up; these differences were not statistically significant.

Table 3 shows these changes in levels of problematic illicit drug use, divided by severity of use categories.

Neither the changes in DUDIT and DUDIT-C scores, nor the proportions of those who had quit using illicit drugs at follow-up, differed statistically by administration mode.

\section{Figure 2a}

\section{Changes in total DUDIT scores by categories of problematic drug use}

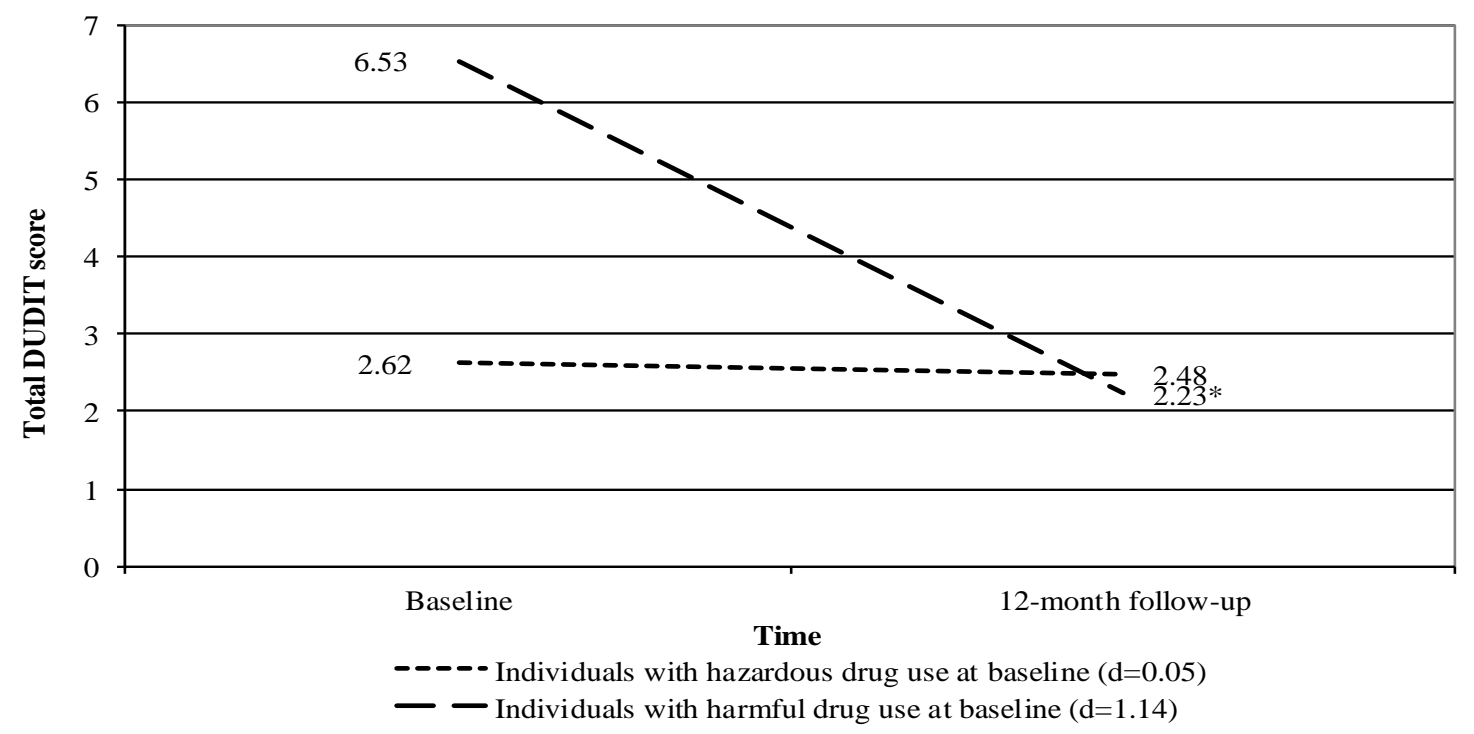

$* p<0.05$ 
Figure 2b

Changes in total DUDIT-C scores by categories of problematic drug use

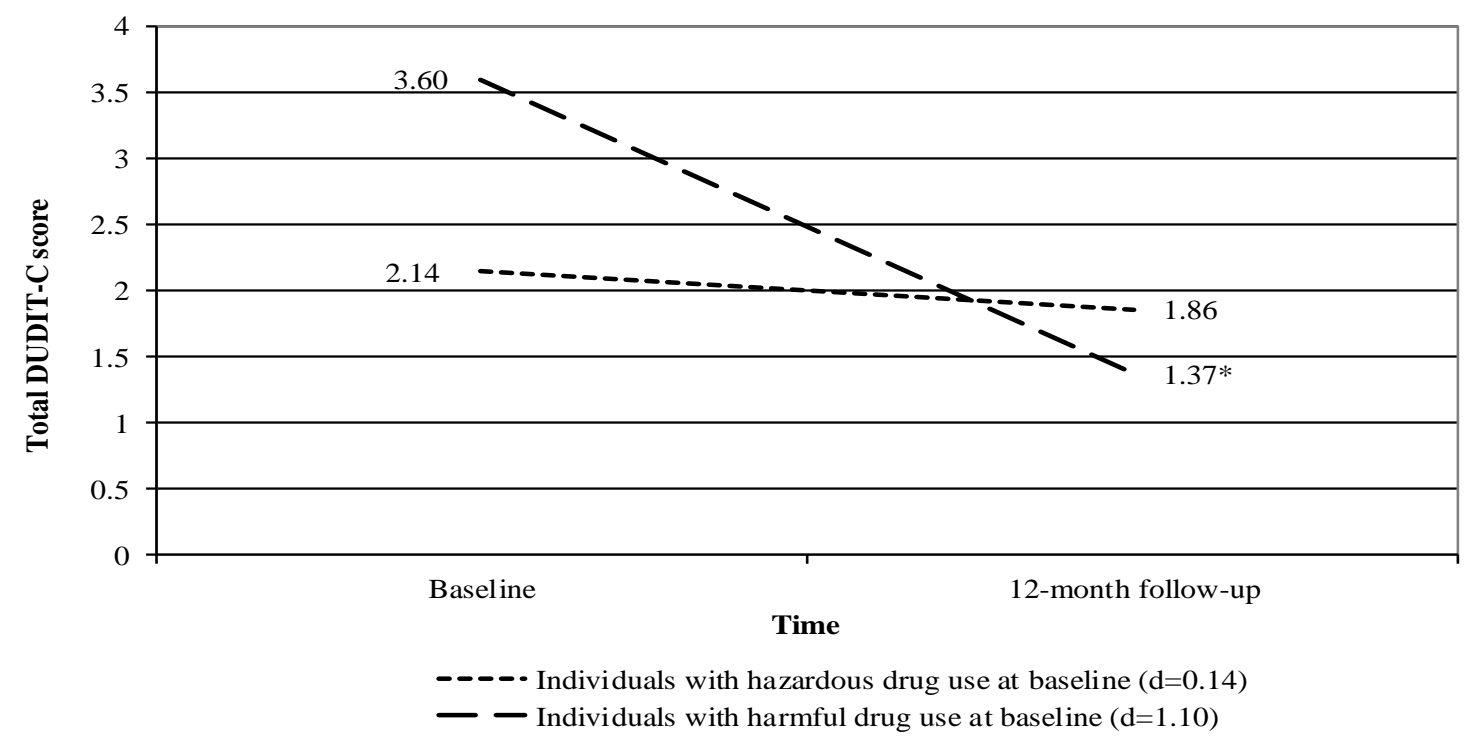

$* p<0.05$

Table 3

Changes in categories of problematic illicit drug use over 12 months $(n=51)$

\begin{tabular}{|c|c|c|c|c|}
\hline \multirow{2}{*}{$\begin{array}{l}\text { Baseline categories of } \\
\text { problematic drug use }\end{array}$} & \multicolumn{3}{|c|}{ Follow-up categories of problematic drug use } & \multirow[b]{2}{*}{ Total } \\
\hline & No drug use & Hazardous drug use & Harmful drug use & \\
\hline Hazardous drug use & $12(57.1 \%)$ & $5(23.8 \%)$ & $4(19.0 \%)$ & $21(100.0 \%)$ \\
\hline Harmful drug use & $19(63.3 \%)$ & $1(3.3 \%)$ & $10(33.3 \%)$ & $30(100.0 \%)$ \\
\hline Total & 31 (60.8\%) & $6(11.8 \%)$ & $14(27.5 \%)$ & $51(100.0 \%)$ \\
\hline
\end{tabular}

\section{Discussion}

In summary, this follow-up study achieved a response rate of $67.9 \%$ and an even distribution of men and women. At the 12-month follow-up, a significant decrease in total AUDIT and DUDIT scores, as well as in AUDIT-C and DUDIT-C scores, was found among both men and women in the sample; the decline was largest in the groups with higher levels of problematic substance use. It was also shown that over one-third of all participants no longer had any problematic alcohol use, and well over half of those with problematic illicit drug use at baseline had stopped using illicit drugs at follow-up. The within-group effect sizes for AUDIT and DUDIT scores and the shorter Cversions varied between 0.29 and 0.69 (Cohen's $d$ ).

No significant differences in response rates or changes in substance use were found between Internet and IVR participants, except that a larger proportion of men who participated in the follow-up via Internet (39.3\%) no longer had problematic alcohol use at follow-up, compared to men who participated via IVR (18.9\%).

Literature about short-term changes in alcohol or illicit drug use in the general population is very sparse. To the best of our knowledge, this study is the only one looking at such changes in both alcohol and illicit drug use. We did find one study that investigated short-term fluctuations in alcohol use in an untreated general population sample from the southern U.S. Study participants, who had either an alcohol disorder diagnosis or a risk for developing such a diagnosis, were interviewed at baseline and followed up six, 12 and 18 months later. In line with our results, this study showed that the average number of drinks per drinking day, as well as recent (past six months) alcohol disorders, decreased during the study period, while six- 
month abstinence increased. However, the results also showed that about $30 \%$ of those who did not meet diagnostic criteria for recent alcohol disorder at baseline later did so (Booth, Fortney, Fortney, Curran, \& Kirchner, 2001). Such an increase in the severity of alcohol use was found for a few participants in our study, but was not discernible on a group level. We also found one study examining changes in methamphetamine and cocaine use among untreated rural stimulant users at six-month intervals over a two-year period. In line with our results, this study also showed a considerable reduction in consumption of both drugs, as well as abstention from both drugs, among one-third of study participants at the twoyear follow-up (Borders et al., 2008).

We would like to emphasize that the short-term changes in problematic substance use found in this study are not equivalent to spontaneous remission, where users change their problematic habits without professional help. In this study, no data were collected on possible use of interventions to reduce problematic substance use, and the participants may or may not have accessed treatment. Previous research has shown that only a small proportion of those with problematic alcohol and drug habits ever seek professional help for their problems (Cunningham, 2005; Cunningham \& Blomqvist, 2006; Cunningham \& Breslina, 2004) and that not many heavy drinkers are actually offered alcohol screening, medical advice or a brief intervention in routine primary care (Denny, Serdula, Holtzman, \& Nelson, 2003; Friedmann, McCullough, Chin, \& Saitz, 2000). However, small proportions of those from the general population who do seek professional help often use several different alcohol treatment services in the same year (Cunningham \& Blomqvist, 2006), and short-term recovery is greater among these than among individuals who do not access treatment (Cunningham, 2005). Irrespective of the reasons for change and the means of accomplishing it, the results from this study show an encouraging overall decrease in alcohol and illicit drug consumption on a general population level; specifically, at follow-up, over one-third no longer had any problematic alcohol use and about $60 \%$ had totally stopped using illicit drugs. For illicit drug use, we can clearly see these changes despite the relatively small sample of 51 problematic users. Given previous research on the small proportion of problematic users who access interventions (Blomqvist, 1996, 1999, 2002; Cunningham, 2005; Cunningham \& Blomqvist, 2006; Cunningham \& Breslina, 2004), it is unlikely that our participants did so to any large extent.

One obvious question about these results is whether the change we saw in problematic substance use was due to the natural fluctuation of such use over time, where we measured the change when participants were in a declining phase. We did observe individuals for whom alcohol and illicit drug use increased at the 12-month follow-up, but these were the exception rather than the rule. It is clear that problematic substance users overall generally decreased their substance consumption, as well as its negative consequences, over a 12-month period. The effect sizes of the changes in our data exceeded the within-group weighted effect sizes of spontaneous remissions, where it has been established that participants have received no professional treatment (Walters, 2000). As noted above, we collected no data regarding any possible treatment received over the 12-month follow-up period.

An additional factor that might also explain the declines we saw in problematic substance use in our general population sample is motivation to change. It may be that the nearly $70 \%$ of our respondents who chose to participate in the 12month follow-up were more motivated than individuals with problematic substance use at large in the population. In an attempt to assess the possible effects of motivation, we compared the effect sizes in this study to effect sizes for control groups in two randomized controlled trials that we have previously conducted among Internet help-seekers from the general population (Sinadinovic, Wennberg, \& Berman, 2012, 2014; Sinadinovic, Wennberg, Johansson, \& Berman, 2014). In these trials, individuals in the general population responded to an online advertisement offering participation in a randomized intervention trial for problematic alcohol or drug use. We might thus have expected their motivation to be higher, overall, than that of the individuals participating in our general sampling pool. The untreated control group in these trials might serve as a sample of motivated individuals who did not receive an intervention, similar to the participants in our general sampling pool except in terms of motivation. In the trial targeting problematic alcohol users, the 12-month effect size for AUDIT changes in the untreated control group was 1.13, and for AUDIT-C it was 1.18 (unpublished), as compared to effect sizes of 0.38 and 0.29 respectively in our general population sampling pool. In the trial targeting problematic drug users, the effect size for DUDIT changes in the untreated control group was 0.69 and for DUDIT-C it was 0.93 (unpublished), as compared with 0.69 for both measures in the currently reported general sampling pool. Effect sizes for alcohol and drug consumption (AUDIT-C and DUDIT-C) were thus higher for untreated control group participants over 12 months, in comparison to individuals in the general population sampling pool. Effect sizes for scores including problem levels (AUDIT and DUDIT) were higher in the untreated control group for alcohol, but not for drugs. It could be that individuals using drugs who were willing to participate in a general population study 12 months after baseline were highly motivated to change their behavior. In sum, participants in our general population sampling pool may have had some motivation to change that would explain the decline in their scores, but the motivation appears to have been lower than that among individuals who chose to participate in randomized controlled trials offering online self-help interventions for problematic alcohol and drug use. Finally, this comparison suggests that using the populationbased effect sizes we report could well have clinical relevance for assessing factors related to change in problematic substance use in different study and treatment contexts.

Two additional potential explanations for the decline in problematic substance use should be noted. Since participants for this follow-up study were recruited based on their high AUDIT and DUDIT scores, all of which were 
above the population mean, the decrease could have resulted from the phenomenon of regression towards the mean. This statistical phenomenon means that the extreme values of a variable measured at one point tend to be closer to the average at the next measurement (Shaughnessy \& Zechmeister, 1990). Indeed, our finding that declines in AUDIT and DUDIT scores were larger for individuals at higher levels of problematic alcohol and drug use could actually support this speculation. In our study, we were not able to control for regression to the mean, since nonproblematic users, whose use would have been expected to increase over time, were not included in the follow-up. A second possible explanation for the general decline in problematic use observed in our study is the corresponding change in total alcohol consumption in Sweden for the studied period. Alcohol consumption per capita among individuals 15 years and older decreased in Sweden from 9.82 liters in 2008 to 9.58 in 2009 (CAN, 2014). According to the total consumption model, changes in total consumption tend to be associated with changes in consumption and alcohol-related problems among the heaviest alcohol users (Schmidt \& Popham, 1978 ), which we observed in our study. Official figures regarding changes in per capita use of illicit drugs do not exist for Sweden. Although several indicators of drug use (i.e., drug seizures by custom agents and police, the number of suspects for drug-related offenses, the number of drugrelated diagnoses and drug-related deaths per capita) have increased between 2008 and 2009 (CAN, 2012), we cannot with any certainty assert that drug use has increased. For this reason, we cannot evaluate our results regarding changes in drug use from a total consumption model perspective.

\section{Limitations}

One limitation of this study is that participants were not asked whether they received any help for their problematic alcohol or drug use during the 12-month follow-up period. The purpose of the study was only to collect data on problematic alcohol and drug use, so no questions on treatment interventions accessed were asked, nor did we ask about reasons for changing problematic habits. We chose not to include questions on treatment interventions because we know that about 70 to $80 \%$ of problematic alcohol users do not seek treatment (Blomqvist, 1996, 2002; Cunningham, 2005; Cunningham \& Blomqvist, 2006; Cunningham \& Breslina, 2004). Also, we did not want to increase the test burden, out of a concern that this might negatively affect response rates at follow-up. Had we included such questions, however, we would have been able to obtain a deeper understanding of our results. Future research should include questions about these factors and should assess any effects on response rates.

Another limitation of this study was that only individuals with problematic alcohol or drug use were followed up. The results clearly indicate that problematic alcohol and drug use change over a short period of time. However, since we did not include individuals without problematic use in the follow-up, we cannot say to what extent nonproblematic alcohol and drug use would remain unchanged over time. Had we included individuals without problematic use, we might also have gained a clearer understanding of the extent to which our results were affected by the regression towards the mean phenomenon.

\section{Future research}

With the above limitations in mind, future research about changes in problematic use should investigate the possibility that therapeutic interventions and/or self-help resources have been accessed by participants and have influenced any observed changes. Possible changes in nonproblematic alcohol and drug use over time should be investigated as well.

Individuals with higher levels of problematic alcohol and drug use decreased their problematic consumption to a greater extent than those with lower levels, but did not dip under the cutoff point for non-problematic use. It would be valuable to continue the follow-up for an additional year, as this would reveal whether this decrease continues until those who began with harmful use and probable dependence actually end up with non-problematic use, or whether problematic use remains constant at the reduced level or increases again.

Another issue that requires further research is the cause of this short-term change; it could be due to the natural fluctuation of problematic substance use over time, or, perhaps, to a combined effect of different formal and informal help resources and relations that people access when changing their problematic substance use. Another interesting question is to what extent our screening contributed to this change. Previous research has shown that screening - with the AUDIT only-leads to a significant decrease in alcohol use, with an effect size of 0.23 (McCambridge \& Day, 2008).

\section{Conclusions}

The results from this study indicate relatively large withingroup effect sizes for problematic substance users from the general population. These results could be useful as normative data that could serve as a benchmark for metaanalysis of uncontrolled studies. In addition, for treatment programs in areas such as social services, where research resources are scarce, our results could offer a basis for longitudinal norms to compare with client groups. Further research in an expanded population study, where both nonproblematic and problematic users would be followed up, might provide a basis for an algorithm that would generate predictive values based on short-term natural change, taking into account severity of use by gender and age. Evaluators in public services could then compare postintervention measures with the short-term change in the general population.

In this study, we identified what might be termed a naturally declining consumption of alcohol and drugs. We thus suggest that the effect sizes found could be used to assess the impact of a treatment method beyond natural decline. Also, treatment outcome studies are generally associated with hypotheses regarding stability of outcome over time; comparing stability of outcomes with population-based naturally occurring changes in 
problematic alcohol and drug use could add valuable information. Uncontrolled treatment studies, where effect sizes cannot be assessed meaningfully, could likewise be reviewed in comparison to population-based data of the kind we have presented. In conclusion, we believe that random population data on short-term changes in problematic substance use, as defined in this study, could constitute an excellent reference point for comparisons with effect sizes in studies investigating specific treatment methods-in particular, effect sizes in uncontrolled treatment studies.

\section{Acknowledgements}

This work was supported by grants provided by the the Swedish National Drug Policy Coordinator (MOB 238/2006:32), the Stockholm Center for Dependency Disorders and Stockholm County Council (ALF project 20110372). The authors acknowledge Jens Pettersson, Interactive Health Group $A B$, technical developer of the technical platform for the study.

\section{References}

Berman, A. H., Bergman, H., Palmstierna, T., \& Schlyter, F. (2005). Evaluation of the Drug Use Disorders Identification Test (DUDIT) in criminal justice and detoxification settings and in a Swedish population sample. European Addiction Research, 11, 22-31.

Berman, A. H., Wennberg, P., \& Källmén, H. (2012). AUDIT och DUDIT - identifiera problem med alkohol och droger [AUDIT and DUDIT - identifying problematic alcohol and drug use]. Stockholm, Sweden: Gothia förlag.

Blomqvist, J. (1996). Paths to recovery from substance misuse: Change of lifestyle and the role of treatment. Substance Use \& Misuse, 31(13), 1807-1852.

Blomqvist, J. (1999). Treated and untreated recovery from alcohol misuse. Environmental influences and perceived reasons for change. Substance Use \& Misuse, 34(10), 1371-1406.

Blomqvist, J. (2002). Recovery with and without treatment: A comparison of resolutions of alcohol and drug problems. Addiction Research \& Theory, 10(2), 119158.

Blomqvist, J., Cunningham, J. A., Wallander, L., \& Collin, L. (2007). Att förbättra sina dryckesvanor - om olika mönster för förändring och om vad vården betyder. [Improving one's drinking habits - on different patterns of change and on the role of alcohol treatment]. Stockholm, Sweden: The Centre for Social Research on Alcohol and Drugs (SoRAD).

Booth, B. M., Fortney, S. M., Fortney, J. C., Curran, G., \& Kirchner, J. E. (2001). Short-term course of drinking in an untreated sample of at-risk drinkers. Journal of Studies on Alcohol, 62(5), 580-588.

Borders, T. F., Booth, B. M., Han, X., Wright, P., Leukefeld, C., Falck, R. S., \& Carlson, R. G. (2008). Longitudinal changes in methamphetamine and cocaine use in untreated rural stimulant users: racial differences and the impact of methamphetamine legislation. Addiction, 103(5), 800-808.

Bush, K., Kivlahan, D. R., McDonell, M. B., Fihn, S. D., \& Bradley, K. A. (1998). The AUDIT alcohol consumption questions (AUDIT-C): an effective brief screening test for problem drinking. Ambulatory Care Quality Improvement Projekt (ACQUIP). Archives of Internal Medicine, 158, 1789-1795.

CAN. (2012). Drogutvecklingen i Sverige 2011. [Drug trends in Sweden 2011]. Stockholm, Sweden: The Swedish Council for Information on Alcohol and Other Drugs.

CAN. (2014). Hur mycket dricker svensken? alkoholkonsumtionen i siffror 2001-2012 [How much does the Swede drinks? - alcohol consumption in numbers 2001-2012]. Stockholm, Sweden: The Swedish Council for Information on Alcohol and Other Drugs.

Cohen, J. (1988). Statistical power analysis for the behavioral sciences (2nd ed.). Hillsdale, NJ, United States: Erlbaum.

Cunningham, J. A. (2005). Short-term recovery from alcohol abuse or dependence: Any evidence of a relationship with treatment use in a general population sample? Alcohol and Alcoholism, 40(5), 419-421.

Cunningham, J. A., \& Blomqvist, J. (2006). Examining treatment use among alcohol-dependent individuals from a population perspective. Alcohol and Alcoholism, 41(6), 632-635.

Cunningham, J. A., \& Breslina, F. C. (2004). Only one in three people with alcohol abuse or dependence ever seek treatment. Addictive Behaviors, 29, 221-223.

Cunningham, J. A., Humphreys, K., Koski-Jännes, A., \& Cordingley, J. (2005). Internet and paper self-help materials for problem drinking: Is there an additive effect? Addictive Behaviors, 30, 1517-1523.

Cunningham, J. A., Wild, T. C., Cordingley, J., van Mierlo, T., \& Humphreys, K. (2009). A randomized controlled trial of an internet-based intervention for alcohol abusers. Addiction, 104, 2023-2032.

Denny, C. H., Serdula, M. K., Holtzman, D., \& Nelson, D. E. (2003). Physician advice about smoking and drinking: are US adults being informed? American Journal of Preventive Medicine, 24, 71-74.

Friedmann, P. D., McCullough, D., Chin, M. H., \& Saitz, R. (2000). Screening and intervention for alcohol problems: A national survey of primary care physicians and psychiatrists. Journal of General Internal Medicine, 15, 84-91.

Hester, R. K., Delaney, H. D., Campbell, W., \& Handmaker, N. (2009). A web application for moderation training: Initial results of a randomized clinical trial. Journal of Substance Abuse Treatment, 37, 266-276.

Koski-Jännes, A., \& Cunningham, J. A. (2001). Interest in different forms of self-help in a general population sample of drinkers. Addictive Behaviors, 26(9), 1-9.

Kypri, K., Hallett, J., Howat, P., McManus, A., Maycock, B., Bowe, S., \& Horton, N. J. (2009). Randomized controlled trial of proactive web-based alcohol screening and brief intervention for University 
students. Archives of Internal Medicine, 169(16), 1508-1514.

McCambridge, J., \& Day, M. (2008). Randomized controlled trial of the effects of completing the Alcohol Use Disorders Identification Test questionnaire on self-reported hazardous drinking. Addiction, 103(2), 241-248.

Raistrick, D., Heather, N., \& Godfrey, C. (2006). Review of the effectiveness of treatment for alcohol problems. London, UK: National Treatment Agency for Substance Misuse.

Riper, H., Kramer, J., Conijn, B., Smit, F., Schippers, G., \& Cuijpers, P. (2009). Translating effective web-based self-help for problem drinking into the real world. Alcoholism: Clinical \& Experimental Research, 33(8), 1-8.

Saunders, J. B., Aasland, O. G., Babor, T. F., De La Fuente, J. R., \& Grant, M. (1993). Development of Alcohol Use Disorders Identification Test (AUDIT): WHO collaborative project on early detection of persons with harmful alcohol consumption - II. Addiction, 88, 791-804.

Schmidt, W., \& Popham, R. E. (1978). The single distribution theory of alcohol consumption. A rejoinder to the critique of Parker and Harman. Journal of Studies on Alcohol, 39(3), 400-419.

Shaughnessy, J. J., \& Zechmeister, E. B. (1990). Research methods in psychology. New York, NY, United States: McGraw-Hill.

Sinadinovic, K., Berman, A. H., Hasson, D., \& Wennberg, P. (2010). Internet-based assessment and selfmonitoring of problematic alcohol and drug use. Addictive Behaviors, 35(5), 464-470.

Sinadinovic, K., Berman, A. H., \& Wennberg, P. (2011). Population screening of risky alcohol and drug use via Internet and Interactive Voice Response (IVR): A feasibility and psychometric study in a random sample. Drug and Alcohol Dependence, 114, 55-60.

Sinadinovic, K., Wennberg, P., \& Berman, A. H. (2012). Targeting problematic users of illicit drugs with internet-based screening and brief intervention: A randomized controlled trial. Drug and Alcohol Dependence, 126(1-2), 42-50.

Sinadinovic, K., Wennberg, P., \& Berman, A. H. (2014). Internet-based screening and brief intervention for illicit drug users: A randomized controlled trial with 12-month follow-up. Journal of Studies on Alcohol and Drugs, 75(2), 313-318.

Sinadinovic, K., Wennberg, P., Johansson, M., \& Berman, A. H. (2014). Targeting individuals with problematic alcohol use via web-based cognitive-behavioral selfhelp modules, personalized screening feedback or assessment only: A randomized controlled trial. European Addiction Research, 20(6), 305-318.

The National Board of Health and Welfare. (2006). Faktaunderlag till Nationella riktlinjer för missbruksoch beroendevård [Data review underlying the Swedish National Guidelines for Treatment of Substance Abuse and Dependence]. Stockholm, Sweden: Author.

The National Board of Health and Welfare. (2007). Swedish National Guidelines for Treatment of
Substance Abuse and Dependence. Stockholm. Sweden: Author.

The Swedish Council on Technology Assessment in Health Care. (2001a). Behandling av alkohol- och narkotikaproblem: En evidensbaserad kunskapssammanställning. [Treatment for alcoholand drug-related problems: An evidence-based literature review.] (Vol. 1). Stockholm, Sweden: Author.

The Swedish Council on Technology Assessment in Health Care. (2001b). Behandling av alkohol- och narkotikaproblem: En evidensbaserad kunskapssammanställning. [Treatment for alcoholand drug-related problems: An evidence-based literature review.] (Vol. 2). Stockholm, Sweden: Author.

Walters, G. D. (2000). Spontaneous remission from alcohol, tobacco, and other drug abuse: Seeking quantitative answers to qualitative questions. American Journal of Drug and Alcohol Abuse, 26(3), 443-460.

WHO. (2007). WHO expert committee on problems related to alcohol consumption. WHO Technical Report Series. Geneva, Switzerland: Author.

WHO. (2009). Global health risks: mortality and burden of disease attributable to selected major risks. (Vol. 2). Geneva, Switzerland: Author. 